\title{
Noise reducing performance of anisotropic diffusion filter and circular median filter in digital images
}

\section{Neha Mehta* and S.V.A.V. Prasad}

Lingaya's Vidyapeeth, Nachauli, Jasana Road, Old Faridabad, Faridabad, Haryana 121002, India Email: neha_28j@rediffmail.com Email: prasad.svav@gmail.com

*Corresponding author

\section{Leena Arya}

Electronics and Communication Department, ITS Engineering College, Greater Noida, U.P., India

Email: dr.leenaarya@its.edu.in

\begin{abstract}
The speckle noise is one of the prominent features found in ultrasound images and it tends to degrade the quality of the image. There is a historic success in the works towards this approach particularly in the medical field related to image de-speckling. In this paper, a scale-space nonlinear anisotropic diffusion process and circular median filter is being combined with un-sharp masking to filter out the speckle noise from the ultrasound images. However, the performance evaluation and the comparison of both the filters are being done at each level of the process by comparing the various parameters of image quality measures. The nonlinear anisotropic diffusion filter has found to be performing better by two decimal point values in comparison to the circular median filter.
\end{abstract}

Keywords: speckle noise; ultrasound images; de-speckling; scale-space nonlinear anisotropic diffusion process; circular median filter; circular median filter; CMF; un-sharp masking; image quality measures.

Reference to this paper should be made as follows: Mehta, N., Prasad, S.V.A.V. and Arya, L. (2020) 'Noise reducing performance of anisotropic diffusion filter and circular median filter in digital images', Int. J. Digital Signals and Smart Systems, Vol. 4, Nos. 1/2/3, pp.171-183.

Biographical notes: Neha Mehta did her MTech and pursuing her PhD from Lingaya's Vidyapeeth. Her areas of interest include medical image processing, wireless and satellite communication. She has also guided five MTech thesis. She has 22 published research papers in various national and international conferences.

S.V.A.V. Prasad is an MTech and $\mathrm{PhD}$ and currently working as a Professor and Dean (academic affairs), Dean (R\&D) and Dean (corporate affairs) in Lingaya's Vidyapeeth. His area of research includes wireless communication, artificial intelligence, brain mapping and so on. He is a reviewer of various national and international journal. He is a fellow member of IEEE and Life Member of ISTE, IETE. 
Leena Arya has a PhD in Wireless Comm. She is having more than 14 years of experience in teaching and research. She has also handled Research Project from Department of Science and Technology, DST, New Delhi as Principal Investigator under Women Scientists Scheme (WOS-A). She is having 25 publications. She is a member of ISTE, ISOC, and IAENG.

\section{Introduction}

Ultrasound technique is a well known low cost and non-invasive procedure found in diagnostic radiology, which is being extensively used. But the presence of speckle noise in the ultrasound images is one of the major drawbacks, which can degrade the quality of the images and also hampers the feature extraction of the image. The ultrasonic speckle (Vanithamani and Umamaheswari, 2010) is the interference effect, generated due to the scattering of ultrasound beam from various tissue homogeneities. Thus, the speckle, does not allow the observer to get the knowledge of fine details of small edges present in the image. The ultrasound images are also known for their low signal to noise ratio values (Singh et al., 2018). Hence, the suppression of speckle noise requires a technique from the field of digital image processing that can substantially filter out the speckle effect from the digital ultrasound images. The application of better filtering technique will definitely help to improve the potential in diagnostic ultrasound imaging. The paper presents the application of nonlinear anisotropic filter and the circular median filter (CMF) along with un-sharp masking effect to better visualise the ultrasound images. However, the comparison on the basis of evaluation metrics is also produced to convey the better results.

The paper is organised as: Section 2 defines the speckle noise with Section 3 covering the previous related works that has been summarised from various literature surveys. Section 4 covers materials and methods used in the proposed work. The flowchart of the proposed methodology is shown in Section 5. In Section 6, the image quality measures are discussed and results and discussion is given in Section 7 while the conclusion is stated in Section 8.

\section{Speckle noise}

The degradation, found in the ultrasound images, is a form of speckle. It is a multiplicative type of noise, which appear in the echo-genic areas of an image (Loizou et al., 2014). The speckle tends to produce a granular appearance, affecting the original image texture. The speckle noise (Benes and Riha, 2011) can be modelled as-

$$
I(i, j)=J(i, j) \cdot m(i, j)+a(i, j)
$$

where

- $I(i, j)$ is a noisy image

- $J(i, j)$ is the intensity of image without speckle

- $M(i, j)$ is a multiplicative component of the speckle noise 
- $A(i, j)$ is a additive component of the speckle noise with $(i, j)$ as spatial coordinates.

However, the ultrasound images are only found to be degraded by multiplicative noise (Benes and Riha, 2011). Thus, the model of speckle noise reduces to:

$$
I(i, j)=J(i, j) * m(i, j)
$$

There have been many techniques given by Hamidi and Daraei (2016) for reduction of speckle and image structure preservation which are being discussed in the following related work.

\section{Related work}

The proposed work has been influenced by various related researches, mentioned as follows.

Weickert et al. (1998) have presented the algorithms that are applicable for reliable nonlinear filtering and in arbitrary dimensions. However, the storage and computation of the procedure is linear. Additive operator-based splitting has been used in the proposed work is equally treated for all the coordinate axes.

Chen et al. (2011) have proposed an anisotropic diffusion method with fixed-point type iteration. They have used multi-grid solver and produced a steady-state solution. Similarly, in $\mathrm{Yu}$ et al. (2011), a time dependent image smoothing is produced for anisotropic diffusion process. The authors have shown a strategy of decreasing gradient threshold with time and signal to noise ratio has been calculated.

An image smoothing, edge-preserving anisotropic diffusion method is given in Lakshmanna and Maheswari (2013), this method also restores the image by using bilateral method. A diffusion model is proposed in this approach, which incorporates variance and local gradient that can preserve fine details present in the image and thus removes the noise. But the researches have shown a limitation of the proposed work for images having impulsive noise. Saleh et al. (2011) has shown a local adjustment of edges, moments and textures present in the image. The researchers have shown the forward- backward mode of diffusion process. This adaptive procedure shows a significant enhancement of features and the local geometry. Halim et al. (2014) describes the application of Perona and Malik anisotropic procedure of diffusion, as a useful de-noising technique for radiographic images. Jameel and Shanavas (2015) have shown the deduction of un sharp part of the images, and have shown the applications in the field of photography. The combination of anisotropic diffusion filter and un sharp masking was used for edge enhancement and noise smoothing in order to give the better results. Kamangar et al. (2016) has incorporated the harmonics function with Weiner filter. Ehsaeyan (2016) has shown curvelet transform for preservation of image edges. In Hamidi and Daraei (2016), various algorithms for pre processing and post processing methods for heart disease have been reviewed. However, the performance of the proposed model is evaluated through peak signal to noise ratio, structural similarity index measurement and the temporal time. 


\section{Materials and methods}

The materials and methods used in the proposed approach include the data, the material and the methods. The proposed work is composed of the original images as data, which are the ultrasound images of a human gallbladder. The 20 sample images have been taken from a locally available ultrasound centres, four of them are randomly selected to test the outcome of the proposed technique. Each image from the selected data set passes through a combination of un-sharp masking filter with nonlinear anisotropic diffusion filter and CMF. They are discussed as follows.

\subsection{Un-sharp masking filter}

As produced by Lakshmanna and Maheswari (2013), the un-sharp masking is a sharpness enhancing classical tool for enhancing the sharpness of an image but it does not enhances the contrast. The un-sharp filter deduces an un-sharp part of the images. It has applications in the field of printing and photography (Jameel and Shanavas, 2015). According to Saleh et al. (2011), if the input image is $I(x, y)$, and $I_{\operatorname{smooth}(x, y)}$ is the smoothen form of $I(x, y)$ then,

$$
U_{S . M}(x, y)=I(x, y)-I_{\text {smooth }}(x, y)
$$

where $U_{S . M}(x, y)$ is an un-sharp mask filtered image.

The un-sharp filter operator is shown as

Figure 1 Un-sharp filtering operation

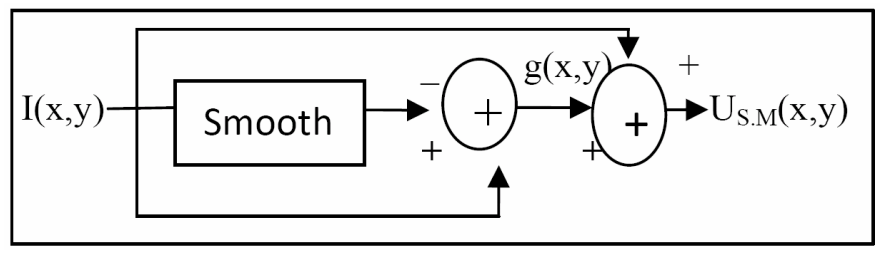

\subsection{Anisotropic diffusion filter}

The process, anisotropic diffusion equation as in Perona and Malik (1990) and Tsiotsios and Petrou (2013) is given as:

$$
\frac{\partial I(x, y, t)}{\partial t}=\operatorname{div}[C(\nabla \cdot I(x, y, t) \|) \cdot \nabla \cdot I(x, y, t) I(x, y, t)]
$$

where $I$ denote the original image, $\nabla(x, y, t)$ is described as the gradient of the image, and $T$ at time, $t$ and $c()$ is a conductance function. For conductance function, $\mathrm{c}$ is chosen as

$$
\lim _{x \rightarrow 0} c(x)=1,
$$

such that the value of diffusion becomes maximum in the regions of uniformity with 


$$
\lim _{x \rightarrow \infty} c(x)=0,
$$

such that the diffusion process is terminated across the edges.

Lakshmanna and Maheswari (2013) have produced a statistical performance for Perona - Malik approach. They have produced the image differentiated density as $I_{n}-I_{s}$, with $I_{n}$, as the neighbouring pixel and pixel $I_{s}$. For the contrast piecewise regions of the image, the neighbour differences are assumed to be small with normally distributed and contains zero-mean. However, if the image region includes the discontinuity among the intensities, then the neighbour differences are not distributed normally.

According to Perona and Malik, the discrete anisotropic diffusion is given by,

$$
I_{i+1}=I_{i}(S)+\frac{\lambda}{|\eta s|} \sum_{q \in \eta s} C k\left(\left|\nabla I_{s, c}\right|\right) \nabla I_{c, q}
$$

where $S$ is the pixel position in a two-dimensional discrete grid, $I$, is a sampled image, $c$ is the conductance function and the gradient threshold parameter is shown by $K$.

The value of $\lambda$ belongs to $(0,1)$ showing the diffusion rate, and four pixel neighbourhood of $\mathrm{S}$ is represented by $\eta_{s}$ spatially. The four neighbourhoods are defined as $\eta_{s}=\{\mathrm{N}, \mathrm{S}, \mathrm{E}, \mathrm{W}\}$ for North N, South S, East E and West W. But they have defined this scheme as low complex computational scheme rather it can preserve many properties in the continuous form (Loizou et al., 2014).

\subsection{Standard paradigm for scale-space strategy}

According to Perona and Malik (1990), the scale space parameter is assumed as a corresponding parameter to the coarser resolution of images. In the standard paradigm, the boundaries were not directly available in the coarse scaled images. However, for two dimensional images the edge junctions were found to be destroyed. Thus, a criterion of scale space tracking is found to be as a solution.

Hence, the description of the scale space paradigm as enunciated by. Perona and Malik in 1990 is found on the basis of causality, immediate localisation leading to sharp boundaries and meaning resolution, piecewise intra-region resolution smoothing. The description of these parameters is as follows.

1 causality: according to the causality property, there should not be a generation of any spurious detail in the images, while processing towards the coarser scales

2 localisation (immediate): according to immediate localisation, the boundaries of the regions should be sharp and must coincide at the same resolution

3 piecewise smoothing: according to piecewise smoothing criteria, the intra region smoothing is preferred at all scales, instead of inter region smoothing.

The scale space representation modified with a new definition along with anisotropic diffusion in Perona and Malik (1990), the authors have discussed the diffusion process. Thus, reducing the effect of linear filter, blurring and dislocation of meaningful edges has also overcome. As produced in Tsiotsios and Petrou (2013), a stopping time, $t$ is estimated and thus it made the art of de-noising as a automatic tool towards the denoising of the image and preserving its edges. 


\section{Conductance}

There were two conductance functions as stated by Perona and Malik in Chen et al. (2001) as

$$
\begin{aligned}
& C_{1}(x)=\exp \left[-\left(\frac{x}{g}\right)^{2}\right] \\
& C_{2}(x)=\frac{1}{1+\left(\frac{x}{g}\right)^{2}}
\end{aligned}
$$

With $g$ as a gradient threshold parameter, and thus controlling the diffusion rate also serving as a soft threshold within the image gradients, attributed towards noise and edges.

The modified conductance functions, later modified in Chen et al. (2001) are given as

$$
\begin{aligned}
& C_{1}(x)=\exp \left[-\left(\frac{x}{g \sqrt{2}}\right)^{2}\right] \\
& C_{2}(x)=\frac{1}{1+\left(\frac{x}{g}\right)^{2}}
\end{aligned}
$$

and

$$
C_{3}(x)=\left\{0.67\left[1-\left(\frac{x}{g \sqrt{5}}\right)^{2}\right]^{2}\right\} \text { for } x \leq \sqrt{5}
$$

It is noticed that, according to $C_{1}$ and $C_{2}$, the flow is found to be continuous and thus smoothes the image. With $C_{3}$ function the flow is seen as descended rapidly and thus the diffusion is stopped and protects the over smoothing of the edges and blurring. However, the function $C_{3}$ is descended fast and prevents the edges within the threshold, $S$. Above the threshold value, the gradients are called as outliers. For any pixel at the coordinates, $(x, y)$ and with iteration, the variance is calculated by its neighbourhood (Patil and Ruikar, 2012).

\subsection{Nonlinear anisotropic diffusion filter}

An improved anisotropic diffusion procedure is known as nonlinear anisotropic diffusion (Patil and Ruikar, 2012). It is the process of nonlinear partial differential equations, that qualitatively removes noise from the images while enhancing the edges and preserving the details. The implementations related to nonlinear anisotropic diffusion methods are found as sensitive to the edge slope parameters (Keeling and Stollberger, 2002). When the nonlinear diffusion filter uses scalar diffusivity, $\mathrm{g}$ with the due image structure, they are called as isotropic with the flux value $(-c \nabla h)$ and is parallel to $\nabla \mathrm{h}$ (Weickert, 1997). However, when the flux is rotated, a nonlinear diffusion tensor is said to be introduced and it is called as nonlinear anisotropic diffusion with $\nabla \mathrm{h}$ as concentration gradient and $c$ 
as conductivity. Jaya and Kumara (2013) have proposed a nonlinear adaptive approach of anisotropic filtering with a choice of selecting edge threshold and conductance function. Greeshma and Kumar (2014) have proposed the improved version of anisotropic filter for efficient removal of noise and thus restore the digital images. The proposed work in this approach uses the modified nonlinear anisotropic diffusion filter with the following features: number of iterations $=3$, value of Diffusion constant has been set to 10 , rate of diffusion $=0.25$.

\subsection{Circular median filter}

The CMF is one of the median filters derived for circular valued data with every pixel values found on the unit radius circle. The arc distance for any image I with values existing on the unit radius circle is given by

$$
F_{m, n}=\arg \min (x \in T) \sum_{i=-v r}^{v r} \sum_{j=-h r}^{h r} D\left(x, I_{m+I, n+j}\right)
$$

\section{Flowchart of proposed methodology}

The proposed methodology is shown through a flow chart in Figure 2.

Figure 2 Flowchart of the proposed methodology

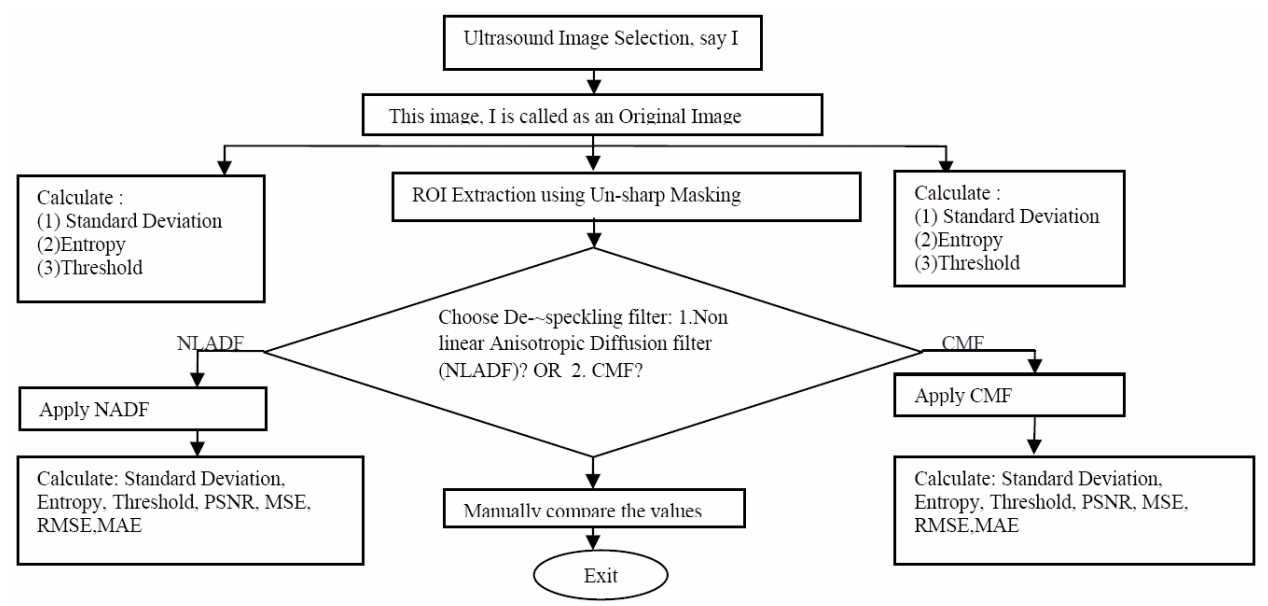

\section{Image quality measurement}

Since, the measurement of the quality of images, have been a crucial task towards image processing methods (Shilpa and Mastani, 2012). The proposed method is tested on the selected set of images. The quality of the ultrasound image is found as mainly affected by the inherent artefact (Flores et al., 2014) called as speckle. An approach has been tried with reduction in speckle while avoiding the over- blurring of edges present in the image. The estimation of quality measures in the proposed work includes. 


\section{Standard deviation}

Standard deviation is a widely used measurement of diversity or variability in statistics (Kumar and Gupta, 2012; Kekre and Patil, 2009). It is a dispersion parameter of variation. The standard deviation is calculated as

$$
S . D(i, j)=\sqrt{\frac{1}{x y-1}} \sum_{(r, C) \in w}\left[g(r, c)-\frac{1}{x y-1} \sum_{(r, c) \in w} g(r, c)\right]^{2}
$$

\section{b Image entropy}

The entropy of an image describes the amount of information present in the image.

The behaviour of entropy (Torres et al., 2015; Li and Wang, 2013) is found less with much darker part in the images. However, the images with zero entropy are called as flat images.

$$
\text { I.E. }=-\sum_{j} P j \log _{2} P_{j}
$$

where $P j$ denotes the probability of difference of two nearby pixels.

c Threshold

The threshold approach (Mingdong et al., 2010; Fu-Song, 2014) is one of the essential parts of image segmentation as produced in Senthilkumaran and Vaithegi (2016) and Kalaiselvi et al. (2017). For any threshold image $t(x, y)$ with $x$ and $y$ as the coordinates of $t$ value point then,

$$
\begin{aligned}
t(x, y)= & \{1, \text { if } q(x, y)>1\} \\
& \{0, \text { if } q(x, y) \leq 0\}
\end{aligned}
$$

And the technique of threshold is defined as

$$
T_{h}=T_{h}[x, y, p(x, y), q(x, y)]
$$

where $p(x, y)$ and $q(x, y)$ are points on gray level image pixels.

d PSNR

PSNR is described as peak signal to noise ratio (Pinki and Mehra, 2016; Pradeep and Balu, 2013) and is the ratio of maximum signal power to the maximum noise power. The value of PSNR should be higher for the good quality of an image. The formula for PSNR is given as

$$
P S N R=10 \log _{10}\left\{\frac{M A X . I}{M S E}\right\}^{2}
$$

e $M S E$

MSE is described as mean square error and is defined as one of the crucial part of image quality metrics (IQMs) (Obulesu and Kishore, 2012). The formula for MSE is given as 


$$
M S E=\sum \frac{[J(x, y)-K(x, y)]^{2}}{M^{*} N}
$$

f RMSE

RMSE is described as root mean square error and is calculated as

$$
R M S E=\sqrt{M S E}
$$

The value of RMSE changes with the variation in error magnitude (Chai and Draxler, 2014).

g $M A E$

MAE is described as mean absolute error, it is defined as maximum absolute difference calculated between the original input image and the degraded image.

MAE is calculated as

$$
M A E=\operatorname{Max}\left(\operatorname{ABS}\left(I_{1}(:)-I_{2}(:)\right)\right.
$$

All the image quality parameters are calculated and are compared as stated in the following results.

\section{Results and discussion}

The sample images (randomly selected four images from the collected database) are allowed to follow the process as given in the proposed flowchart and this work compares the result of nonlinear anisotropic diffusion filter, and the CMF in the terms of standard deviation, entropy and threshold against the similar parameters of original images and the ROI extracting un-sharp masking filter. However, the performance analysis of the proposed method has been evaluated through PSNR, MSE, RMSE, and MAE values of both nonlinear anisotropic filter and CMF. These results are illustrated in Tables 1, 2, 3, 4, 5 and 6 as shown below.

Table 1 Parametric values for original images

\begin{tabular}{lccc}
\hline Image no. & Standard deviation & Entropy & Threshold \\
\hline Image 1 & 0.24841 & 6.0224 & 0.3764 \\
Image 2 & 0.21971 & 7.1833 & 0.4274 \\
Image 3 & 0.27985 & 6.6497 & 0.3686 \\
Image 4 & 0.25243 & 7.4542 & 0.5137 \\
\hline
\end{tabular}

Table 2 Parametric values achieved through un-sharp masking

\begin{tabular}{lccc}
\hline Image no. & Standard deviation & Entropy & Threshold \\
\hline Image 1 & 0.26512 & 3.73061 & 0.356863 \\
Image 2 & 0.20852 & 4.2195 & 0.30196 \\
Image 3 & 0.32229 & 4.18497 & 0.34509 \\
Image 4 & 0.29489 & 4.22803 & 0.388235 \\
\hline
\end{tabular}


Table 3 Parametric values achieved through NLADF

\begin{tabular}{lccc}
\hline Image no. & Standard deviation & Entropy & Threshold \\
\hline Image 1 & 55.55796 & 4.40911 & 0.27451 \\
Image 2 & 64.89698 & 4.6086 & 0.3411 \\
Image 3 & 68.49614 & 4.70430 & 0.29411 \\
Image 4 & 68.78409 & 4.51073 & 0.23921 \\
\hline
\end{tabular}

Table 4 Parametric values achieved through CMF

\begin{tabular}{lccc}
\hline Image no. & Standard deviation & Entropy & Threshold \\
\hline Image 1 & 54.9805 & 0.98324 & 0.49803 \\
Image 2 & 64.8969 & 4.60864 & 0.34117 \\
Image 3 & 68.1079 & 0.99243 & 0.49803 \\
Image 4 & 68.7840 & 4.51073 & 0.23921 \\
\hline
\end{tabular}

Table 5 Performance achieved through NLADF

\begin{tabular}{lcccc}
\hline Image no. & $P S N R$ & $M S E$ & $R M S E$ & $M A E$ \\
\hline Image 1 & 58.4731 & 0.0931 & 0.3052 & 0.1777 \\
Image 2 & 57.0378 & 0.1296 & 0.3600 & 0.2852 \\
Image 3 & 56.2914 & 0.1539 & 0.3923 & 0.2750 \\
Image 4 & 54.5717 & 0.2287 & 0.4782 & 0.4062 \\
\hline
\end{tabular}

Table 6 Performance achieved through CMF

\begin{tabular}{lcccc}
\hline Image no. & $P S N R$ & $M S E$ & $R M S E$ & $M A E$ \\
\hline Image 1 & 58.4578 & 0.0935 & 0.3057 & 0.1782 \\
Image 2 & 57.0378 & 0.1296 & 0.3600 & 0.2852 \\
Image 3 & 56.2914 & 0.1539 & 0.3923 & 0.2750 \\
Image 4 & 54.5717 & 0.2287 & 0.4782 & 0.4062 \\
\hline
\end{tabular}

The above stated values have been found, and it is seen that, as compared to the original images, the information entropy is slightly decreased in un-sharp masking and anisotropic diffusion filter. However, it is found more decreased in the case of CMF. The value of threshold is found least in anisotropic diffusion filter and maximum in the case of CMF. But, the value of standard deviation is found least with the un-sharp masking process. The value of PSNR, MSE, RMSE and MAE are nearly same for both nonlinear anisotropic diffusion filter and the CMF.

\section{Conclusions}

The implementation of CMF and the nonlinear anisotropic diffusion filter has retrieved that there is only two point difference between the comparable values of peak signal to noise ratio, MSE values, RMSE values and the values of MAE. CMFs being with increasing value of threshold and decreased value of information entropy, it is less 
preferred for the de-noising of ultrasound images. And thus, the nonlinear anisotropic diffusion filter is the suggested filter for better performance.

\section{References}

Benes, R. and Riha, K. (2011) 'Noise reduction in medical ultrasound images', Elektrorevue Journal, Vol. 2, No. 3, pp.1-8.

Chai, T. and Draxler, R.R. (2014) 'Root mean square error (RMSE) or mean absolute error (MAE)? - Arguments against avoiding RMSE in the literature', Geoscientific Model Development, Vol. 7, pp.1247-1250.

Chao, S.M., Tsai, D.M., Lee, Y.H., Chiu, W.Y. and Fang, C.H. (2010) 'Anisotropic diffusion-based detail-preserving smoothing for image restoration', IEEE International Conference on Image Processing, DOI:10.1109/ICIP.2010.5653571.

Chen, D., MacLachlan, S. and Kilmer, M. (2011) 'Iterative parameter-choice and multigrid methods for anisotropic diffusion denoising', SIAM Journal of Scientific Computing, Vol. 33, No. 5, pp.2972-2994.

Chen, Y., Barcelos, C.A.Z. and Mair, B.A. (2001) 'Smoothing and edge detection by time-varying coupled nonlinear diffusion equations', Computer Vision and Image Understanding, Vol. 82, No. 2, pp.285-100, Elsevier.

Ehsaeyan, E. (2016) 'An efficient curvelet framework for denoising images', IJE TRANSACTIONS $B$, Vol. 29, No. 8, pp.1094-1102.

Flores, W.G., Pereira, W.C.D. and Infantosi, A.F.C. (2014) 'Breast ultrasound despeckling using anisotropic diffusion guided by texture descriptors', Ultrasound in Med. \& Biol., Vol. 40, No. 11, pp.1-13.

Fu-Song, X. (2014) 'Survey over image thresholding techniques based on entropy', International Conference on Information Science, Electronics and Electrical Engineering (ISEEE).

GitHubInc. (US) (2014) 'Circle median filter', IEEE International Conference on Image Processing, DOI:10.1109/ICIP.2010.5653571 [online] https://github.com/mstorath/ CircleMedianFilter.

Greeshma, P.V. and Kumar, R.R. (2014) 'Denoising algorithm based on anisotropic diffusion with vector median filtering', International Journal of Engineering Research \& Technology, Vol. 3, No. 2, pp.44-7.

Halim, S.A., Razak, R.A., Ibrahim, A. and Manurung, Y.H.P. (2014) 'Application of Perona Malik anisotropic diffusion on digital radiographic image', AIP Conference Proceedings, No. 1605, pp.46-51.

Hamidi, H. and Daraei, A. (2016) 'Analysis of pre processing and post processing methods and using data mining to diagnose heart diseases', IJE TRANSACTIONS A, Vol. 29, No. 7, pp.921-930.

Jameel, S.A. and Shanavas, M. (2015) 'Implementation of improved Gaussian filter algorithm for retinal fundus images', International Journal of Computer Applications, Vol. 132, No. 8, pp.1-4.

Jaya, V.L. and Kumara, R.G. (2013) 'IEM: a new image enhancement metric for contrast and sharpness measurements', International Journal of Computer Applications, Vol. 79, No. 9, pp.1-9.

Kalaiselvi, T., Nagaraja, P. and Indhu, V. (2017) 'A comparative study on thresholding techniques for gray image binarization', International Journal of Advanced Research in Computer Science, Vol. 8, No. 7, pp.1168-1172.

Kamangar, M.H., Karami, M.R., Mollaeia and Ghaderi, R. (2016) 'Determination of fiber detection in high angular resolution diffusion images using spherical harmonics functions and Weiner filter', IJE TRANSACTIONS C, Vol. 29, No. 3, pp.328-336. 
Keeling, S.L. and Stollberger, R. (2002) 'Nonlinear anisotropic diffusion filtering for multiscale edge enhancement', Research Gate (Online), DOI: 10.1088/0266-5611/18/1/312.

Kekre, H.B. and Patil, K. (2009) 'Standard deviation of mean and variance of rows and columns of images for CBIR', International Journal of Computer and Information Engineering, Vol. 3, No. 3, pp.570-73.

Kumar, V. and Gupta, P. (2012) 'Importance of statistical measures in digital image processing', International Journal of Emerging Technology and Advanced Engineering, Vol. 2, No. 8, pp.56-62.

Lakshmanna, M. and Maheswari, A. (2013) 'Modified classical un-sharp masking algorithm', International Journal of Advanced Research in Computer Science and Software Engineering, Vol. 3, No. 9, pp.271-76.

Li, R. and Wang, H. (2013) 'A novel approach of calculating information entropy in information extraction', International Journal of Database Theory and Application, Vol. 6 No. 5, pp.45-52.

Loizou, C.P., Theofanous, C., Pantziaris, M. and Kasparis, T. (2014) 'Despeckle filtering software toolbox for ultrasound imaging of the common carotid artery', Computer Methods and Programs in Biomedicine, Vol. 114, No. 1, pp.109-124.

Mingdong, L., Ding, P. and Ziyang, X. (2010) 'Maximum entropy thresholding segmentation research in 3D images', 2nd International Conference on Advanced Computer Control (ICACC).

Obulesu, M. and Kishore, V.V. (2012) 'A new approach for sharpness and contrast enhancement of an image', International Journal of Advanced Research in Computer Engineering \& Technology, Vol. 1, No. 4, pp.530-535.

Patil, V.D. and Ruikar, S.D. (2012) 'Wavelet based image enhancement using nonlinear anisotropic diffusion', International Journal of Advanced Research in Computer Science and Software Engineering, Vol. 2, No. 3, pp.158-162.

Perona, P. and Malik, J. (1990) 'Scale-space and edge detection using anisotropic diffusion', IEEE Transactions on Pattern Analysis and Machine Intelligence, Vol. 12, No. 7, pp.629-639.

Pinki and Mehra, R. (2016) 'Estimation of the image quality under different distortions', International Journal of Engineering and Computer Science, Vol. 5, No. 7, pp.17291-17296.

Pradeep, M. and Balu, S. (2013) 'Comparative study of PSNR evaluation for enhancing the de-noisy image', International Journal of Advanced Research in Computer Science and Software Engineering, Vol. 3, No. 2, pp.202-206.

Saleh, H., Ahmed, S. and Nordin, M.J. (2011) 'Improving diagnostic viewing of medical images using enhancement algorithms', Journal of Computer Science, Vol. 7, No. 12, pp.1831-1838.

Senthilkumaran, N. and Vaithegi, S. (2016) 'Image segmentation by using thresholding techniques for medical images', Computer Science \& Engineering: An International Journal (CSEIJ), Vol. 6, No. 1, pp.1-13.

Shilpa, K. and Mastani, S.A. (2012) 'Comparison of image quality matrics', International Journal of Engineering Research and Technology, Vol. 1, No. 4, pp.1-6.

Singh, P., Mukundan, R. and Ryke, R.D. (2018) 'Texture based quality analysis of simulated synthetic ultrasound images using local binary patterns', Journal of Imaging, MDPI, Vol. 4, No. 1, pp.1-13.

Torres, E., Garces, Y., Pereira, O. and Morales, R.R. (2015) 'Behavior study of entropy in a digital image through an iterative algorithm of the mean shift filtering', Research Gate (online), DOI: $10.14810 / \mathrm{ijscmc} .2015 .4301$.

Tsiotsios, C. and Petrou, M. (2013) 'On the choice of the parameters for anisotropic diffusion in image processing', Pattern Recognition, Vol. 46, No. 5, pp.1369-1381, Elsevier.

Vanithamani, R. and Umamaheswari, G. (2010) 'Performance analysis of filters for speckle reduction in medical ultrasound images', International Journal of Computer Applications, Vol. 12, No. 6, pp.23-27. 
Weickert, J. (1997) 'A review of nonlinear diffusion filtering', Scale Space Theory in Computer Vision_Lecture Notes in Computer Science, No. 1252, pp.3-28, Springer Berlin.

Weickert, J., Romeny, B.M.H. and Viergever, M.A. (1998) 'Efficient and reliable schemes for nonlinear diffusion filtering', IEEE Transactions on Image Processing, Vol. 7, No. 3, pp.398-410.

Witkin, A.P. (1983) Scale Space Filtering, pp.1020-1022, Fairchild Laboratory for Artificial Intelligence Research book, West Germany.

Yu, X., Wu, C., Jia, T. and Chen, S. (2011) 'A time-dependent anisotropic diffusion image smoothing method', Second International Conference on Intelligent Control and Information Processing (ICICIP). 